University of Nebraska - Lincoln

DigitalCommons@University of Nebraska - Lincoln

\title{
Investigation of electrical transport in hydrogenated multiwalled carbon nanotubes
}

\author{
Adam L. Friedman \\ US Naval Research Laboratory, adam.friedman.ctr@nrl.navy.mil \\ Hyunkyung Chun \\ Northeastern University \\ Don Heiman \\ Northeastern University \\ Yung Joon Jung \\ Northeastern University, jungy@coe.neu.edu \\ Latika Menon \\ US Naval Research Laboratory
}

Follow this and additional works at: https://digitalcommons.unl.edu/usnavyresearch

Part of the Operations Research, Systems Engineering and Industrial Engineering Commons

Friedman, Adam L.; Chun, Hyunkyung; Heiman, Don; Jung, Yung Joon; and Menon, Latika, "Investigation of electrical transport in hydrogenated multiwalled carbon nanotubes" (2011). U.S. Navy Research. 8.

https://digitalcommons.unl.edu/usnavyresearch/8

This Article is brought to you for free and open access by the U.S. Department of Defense at DigitalCommons@University of Nebraska - Lincoln. It has been accepted for inclusion in U.S. Navy Research by an authorized administrator of DigitalCommons@University of Nebraska - Lincoln. 


\title{
Investigation of electrical transport in hydrogenated multiwalled carbon nanotubes
}

\author{
Adam L. Friedman ${ }^{\mathrm{a}, \mathrm{b}, *}$, Hyunkyung Chun ${ }^{\mathrm{c}}$, Don Heiman ${ }^{\mathrm{a}}$, Yung Joon Jung ${ }^{\mathrm{c}}$, Latika Menon ${ }^{\mathrm{a}}$ \\ ${ }^{a}$ Department of Physics, Northeastern University, Boston, MA 02115, USA \\ b Code 6876, US Naval Research Laboratory, Washington, DC 20375, USA

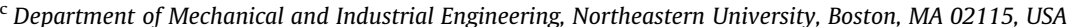

\section{A R T I C L E I N F O}

Article history:

Received 30 November 2010

Accepted 2 December 2010

Available online 9 December 2010

Keywords:

Carbon nanotubes

Electrical transport

Ferromagnetism

Carbon

\begin{abstract}
A B S T R A C T
Highly disordered multiwalled carbon nanotubes of large outer diameter $(\sim 60 \mathrm{~nm})$ fabricated by means of chemical vapor deposition process inside porous alumina templates exhibit ferromagnetism when annealed in a $\mathrm{H}_{2} / \mathrm{Ar}$ atmosphere. In the presence of an applied magnetic field, there is a transition from positive to negative magnetoresistance. The transition may be explained in terms of the Bright model for ordered and disordered carbon structures. Additionally, temperature dependent electrical transport experiments exhibit a zero-bias anomaly at low temperature.
\end{abstract}

(c) 2010 Elsevier B.V. All rights reserved.
There are many reports on the electrical transport properties of carbon nanotubes (CNTs). Most of them exhibit a zero-bias anomaly (ZBA) associated with Luttinger liquid (LL) behavior in highly ordered CNTs [1,2]. However, some have suggested that apart from a LL explanation, for quasi-ballistic single electron junctions in disordered conductors, a ZBA can arise due to either electron or plasmon scattering near the tunnel barrier and finite size effects or due to extremely high resistance contacts or transmission lines [3-6]. However, there are very few studies reported to date on the transport properties of disordered multiwall CNTs (MWCNTs). Initial measurements indicate that the electrical transport properties of disordered MWCNTs can be explained in terms of weak localization theory [7,8]. Weak localization or Anderson localization also predicts that in the presence of an applied magnetic field a sufficiently disordered nanotube will exhibit negative magnetoresistance (MR) [9]. However, the magnetotransport behavior, even for well-ordered nanotubes, appears to be dominated by weak localization effects rather than the LL [9]. This indicates that an applied magnetic field effectively destroys the LL. However, in order to observe such weak localization effects, the temperature must be sufficiently low. In most previous studies, negative MR was only observed for temperatures below $5 \mathrm{~K}$ for ordered CNTs [9].

In this work, we report results of electrical transport measurements on highly disordered MWCNTs fabricated by means of chemical vapor deposition (CVD) inside nanoporous alumina

\footnotetext{
* Corresponding author at: Code 6876, US Naval Research Laboratory, Electronic Materials and Devices, 4555 Overlook Avenue SW, Washington, DC 20375, USA. Tel.: +1 2024044573 .

E-mail address: adam.friedman.ctr@nrl.navy.mil (A.L. Friedman).
}

templates. Earlier we reported that ferromagnetism in such nanotubes can be induced by annealing in hydrogen [12]. The nature of this ferromagnetism has been discussed in the literature [13-16]. In this work, we study temperature dependent transport properties of ferromagnetic nanotubes. We show that at low temperatures and in zero magnetic field, the nanotubes exhibit a ZBA. MR measurements for the ferromagnetic MWCNTs show positive MR at low temperatures, in some cases up to $\sim 40 \mathrm{~K}$, beyond which they exhibit negative MR. Such a transition in MR is not seen in non-ferromagnetic MWCNTs annealed without hydrogen. This transition may be attributed to increased disorder and may possibly be explained in terms of the Bright model [10].

The MWCNTs are synthesized inside nanoporous alumina templates fabricated by anodization of aluminum foil [17]. The template acts as a deposition substrate for the MWCNTs yielding nanotubes with outer diameter ( $\sim 60 \mathrm{~nm}$ for our study) corresponding with the pore diameter of the template. MWCNTs are synthesized without catalyst in the templates in a CVD process at $660{ }^{\circ} \mathrm{C}$ with acetylene acting as the precursor gas. By adjusting the CVD time, we control the inner diameter of the MWCNTs. Here, we make MWCNTs with four different inner diameters using CVD times of $60,75,90$, and $100 \mathrm{~min}$. The right inset of Fig. 1 shows the inner diameter of MWCNTs as a function of CVD time.

The nanotubes are then removed from the templates by dissolving the alumina in $15 \%$ sulfuric acid heated to $100{ }^{\circ} \mathrm{C}$. The acid is removed from the solution and replaced with $99.5 \%$ ethanol. The nanotubes are then dispersed in the solution by sonicating for $3 \mathrm{~s}$ in an ultrasonic bath. TEM studies show that the tubes are polycrystalline and extremely disordered (left inset of Fig. 1). However, the nanotubes retain the SP2 bonding characteristic of a carbon nanotube, as evidenced by XPS studies [12]. A drop of nanotube solution is 


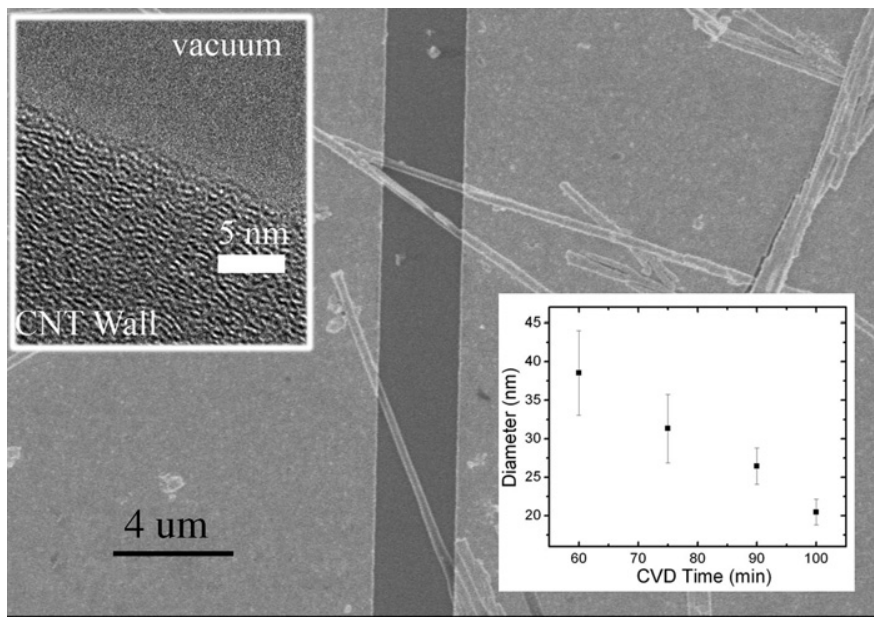

Fig. 1. Scanning electron microscope (SEM) image of a disordered, multiwall carbon nanotubes device. Transmission electron microscope (TEM) image (left inset) of the disordered nanotube, and (right inset) graph of inner diameter versus chemical vapor deposition (CVD) time.

deposited onto a $\mathrm{Si} / \mathrm{SiO}_{2}$ substrate and then the substrate is heated slightly until the ethanol is evaporated out resulting in a welldispersed, sparse film of nanotubes on the $\mathrm{Si} / \mathrm{SiO}_{2}$. The chips are then annealed in a tube furnace containing equal parts $\mathrm{Ar}$ and $\mathrm{H}_{2}$ at $800{ }^{\circ} \mathrm{C}$, which induces ferromagnetism [12]. For comparison, some chips were annealed in Ar only. It was found that annealing also lowers the contact resistances. Un-annealed devices resulted in 2-probe resistances of 1-100 $\mathrm{M} \Omega$, while those annealed had 2-probe resistances of 4-60 k $\Omega$. Lower annealing temperatures were tried, however, this did not successfully lower the contact resistance. Moreover, the temperature necessary to make and break $\mathrm{C}-\mathrm{H}$ bonds is estimated to be $600{ }^{\circ} \mathrm{C}$ [18]. We are well above this limit. Furthermore, TEM studies on the annealed tubes show that the polycrystalline and disordered nature of the tubes is negligibly altered. We found that the structure of the un-annealed tubes, $\mathrm{H}_{2} / \mathrm{Ar}$ annealed, and $\mathrm{Ar}$ annealed tubes to be identical. This is further supported by studies that consider $800^{\circ} \mathrm{C}$ to be too low for significant graphitization [11]. Therefore, improved crystal ordering and enhanced transport effects as a result of annealing are unlikely.

For electrical transport measurements, 2-probe devices are made by photolithography. We also tested 4-probe devices and found that the contact resistances are low and those 2-probe devices, being easier to fabricate, will suffice for our experiments. The gap between electrodes is approximately $3 \mu \mathrm{m}$. The nanotubes are disordered to the extent that they do not contain continuous walls over this measuring distance. $\mathrm{Cr} / \mathrm{Au}$ contacts of 5/60 nm are deposited in an electron beam evaporator. The photolithography method makes many devices simultaneously on a single chip. Suitable devices were chosen where only a few nanotubes bridge the contacts. We found almost no difference in conductivity between devices where 1,2 , or 3 nanotubes bridged the contacts. Fig. 1 displays a scanning electron microscopy (SEM) image of one such device. For MR measurements, we use a 2 terminal DC set up. Measurements are made using a $14 \mathrm{~T}$ cryo-free superconducting magnetic system and a variable temperature cryostat insert. The magnetic field is applied normal to the long axis of the nanotube.

Fig. 2 shows the resistance versus temperature, $R(T)$, of some typical hydrogen annealed and argon-only annealed nanotubes for four different tube diameters. We see that for all samples, the resistance versus temperature curve is punctuated by a "knee" at approximately $14 \mathrm{~K}$. Beyond this knee, the resistance increases dramatically. We made several batches of devices, and the behavior was consistent across all devices made at different times. The position of the knee (which is consistently at about 13 or $14 \mathrm{~K}$ for all samples studied) is independent of all other observed behavior in the nanotubes.

The conductance as a function of bias voltage, $G(V)$, for a typical hydrogen annealed sample and an argon-only annealed sample are also shown in Fig. 2. On decreasing temperature, the conductivity at $V=0$ decreases toward $G=0$. One possible explanation for the ZBA is the Luttinger model. LL theory predicts near-ohmic behavior near room temperature with the development of a singularity in the conductance curve as the temperature decreases, which at low enough temperatures eventually become a conductance gap. We can see that, indeed, our samples show the formation of the predicted conductance gap, while approaching ohmic behavior at higher temperatures; all the samples tested showed this behavior. The depth of the conductance singularity and the temperature at which the sample becomes ohmic appears to be independent of inner diameter of the CNT. Also, this behavior persisted for both $\mathrm{Ar}$ and $\mathrm{H}_{2}$ annealed devices.

The strongly correlated electron system of a LL results in a power law depends on the density of states as a function of energy which in turn leads to a differential conductance, $G_{L L}=\mathrm{d} I / \mathrm{d} V$, given by $[1,2]$

$$
G_{L L}(V, T)=A T^{\alpha}\left|\Gamma\left(z+\frac{1}{2}\right)\right|^{2} \sinh \left(\frac{x}{2}\right)\left[\frac{1}{2} \operatorname{coth}\left(\frac{x}{2}\right)-\frac{1}{\pi} \operatorname{Im} \Psi\left(z+\frac{1}{2}\right)\right]
$$

where

$$
z=1+\alpha / 2+i x / 2 \pi
$$

$x=\eta e V / k_{B} T$

Thus, the model has three parameters: $\alpha, \eta$, and $A$. $A$ is a constant and $\eta$ accounts for voltage division of the nanotube at the electrical contacts, i.e. tunnel barriers. It should be 0.5 for two contacts. The value of the exponential scaling factor $\alpha$ strongly depends on the type of electron tunneling in the device. There are two cases:

$$
\begin{aligned}
& \alpha_{\text {end }}=\frac{1}{4 N}\left(\frac{1}{g}-1\right) \\
& \alpha_{\text {bulk }}=\frac{1}{8 N}\left(\frac{1}{g}+g-2\right)
\end{aligned}
$$

Here, $N$ refers to the number of layers, or conducting channels and $g$ is the so-called Luttinger interaction parameter that measures the strength of the interaction between electrons. The resistance (and the $\mathrm{LL}$ ) is contained at the points of contact between the nanotube and the electrical contact. So, the first case is that an electron tunnels from the contact into the LL from the end $\left(\alpha_{\text {end }}\right)$, in the direction along the length of the nanotube. The second case is that an electron tunnels from the contact into the LL from the side $\left(\alpha_{\text {bulk }}\right)$, at some angle from the length of the nanotube. In any case, for a strongly correlated system such as this, we expect $g<1$. For $g=1$, we would have the case of a non-interacting Fermi liquid. For the case of $g \ll 1$, we would have very strong repulsive electronelectron interactions and the material would become localized. Based on this, one can make a theoretical prediction for $\alpha$. Graugnard et al. [2] predicted a range of 0.2-0.6. Bockrath et al. [1] predicted that $\alpha_{\text {end }}=0.65$ and $\alpha_{\text {bulk }}=0.24$. Indeed, the conductance in our samples decreases like $T^{\alpha}$ and is consistent with LL model and with previous studies [1,2]. A detailed analysis of various samples and the calculated $\alpha$ are provided in Table 1 .

Another prediction of the Luttinger model, from Eq. (1), is that a plot of $G(V, T) /\left(G_{0} / T^{\alpha}\right)$ versus $e V / k_{B} T$ should show scaling where the data collapses onto a universal curve. Our results for an $\mathrm{Ar}$ annealed sample and $\mathrm{a}_{2}$ annealed sample are displayed in Fig. 3. The conductance data was fit to Eq. (1) by varying the parameters $A, \alpha$, and $\eta$ to the lowest temperature data set. We see that the Luttinger model provides a reasonably good fit to the data. However, there are significant deviations from the predicted universal curve as also reported in 

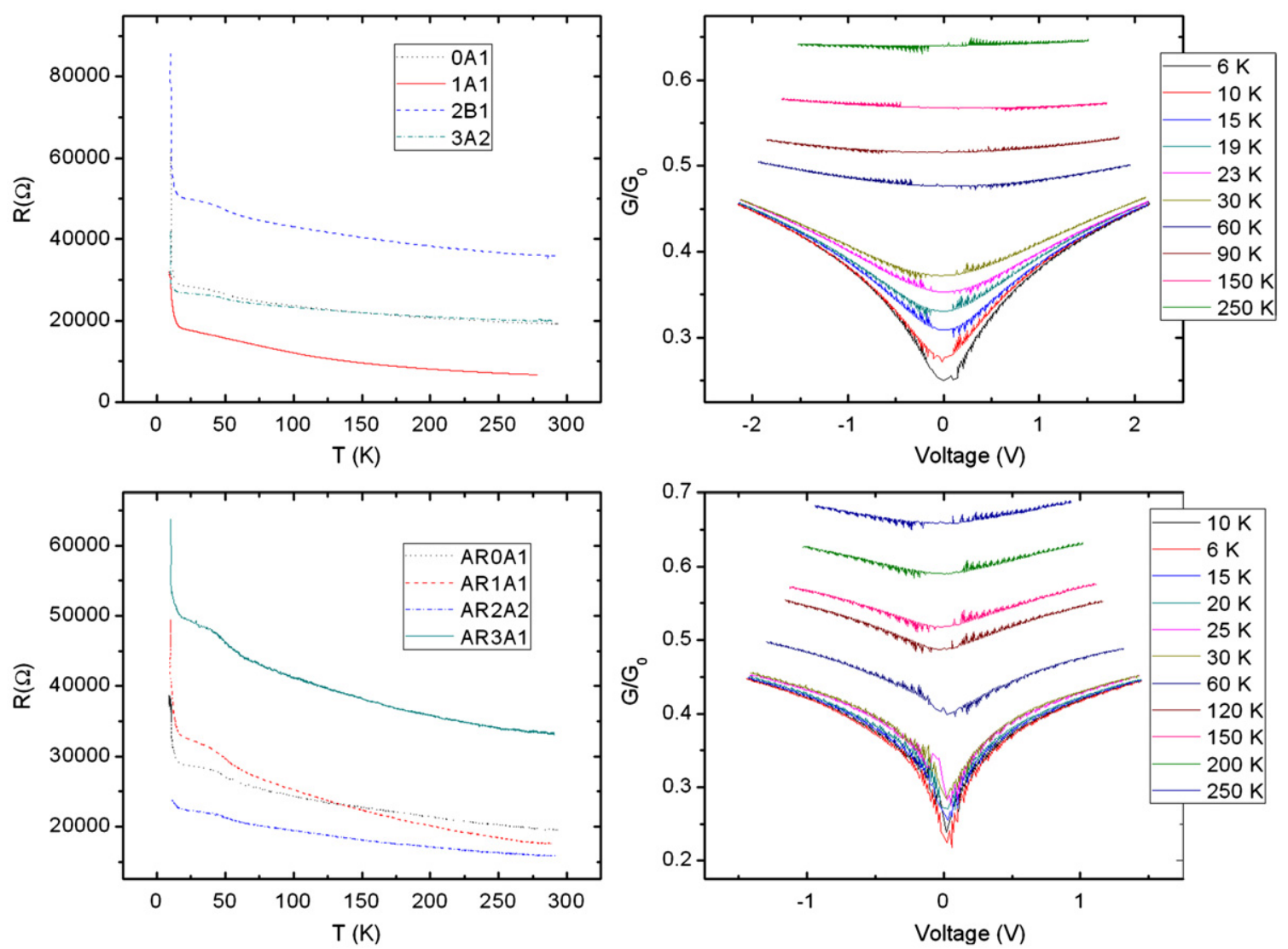

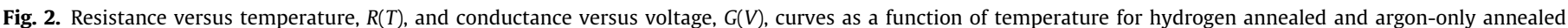

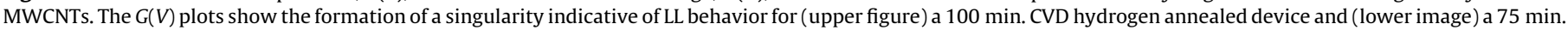
argon annealed device.

Table 1

Luttinger fitting parameters for hydrogen annealed MWCNTs device.

\begin{tabular}{lllllll}
\hline Sample & Annealing & $\begin{array}{l}\text { CVD } \\
\text { time } \\
(\mathbf{m i n})\end{array}$ & $\begin{array}{l}\text { Meas. } \\
\boldsymbol{\alpha}\end{array}$ & $\begin{array}{l}\text { Scaled } \\
\boldsymbol{\alpha}\end{array}$ & $\begin{array}{l}\text { Scaled } \\
\boldsymbol{\eta}\end{array}$ & $\boldsymbol{A}$ \\
& & & & & \\
\hline 0A1 & $\mathrm{H}_{2} / \mathrm{Ar}$ & 60 & 0.282 & 0.833 & 0.00718 & 0.286 \\
$1 \mathrm{~A} 1$ & $\mathrm{H}_{2} / \mathrm{Ar}$ & 75 & 0.420 & 0.683 & 0.00871 & 0.227 \\
$2 \mathrm{~B} 1$ & $\mathrm{H}_{2} / \mathrm{Ar}$ & 90 & 0.171 & 0.768 & 1.694 & 0.0546 \\
3A3 & $\mathrm{H}_{2} / \mathrm{Ar}$ & 100 & 0.221 & 0.784 & 0.00454 & 0.407 \\
$\mathrm{AR0A2}$ & $\mathrm{Ar}$ & 60 & 0.119 & 0.843 & 3.439 & 0.229 \\
$\mathrm{AR} 1 \mathrm{~A} 1$ & $\mathrm{Ar}$ & 75 & 0.277 & 0.821 & 0.0223 & 0.311 \\
$\mathrm{AR2A1}$ & $\mathrm{Ar}$ & 90 & 0.158 & 0.846 & 1.287 & 0.142 \\
$\mathrm{AR} 3 \mathrm{~A} 1$ & $\mathrm{Ar}$ & 100 & 0.168 & 0.847 & 0.0192 & 0.278 \\
\hline
\end{tabular}

literature by other groups [1,2], in particular, the voltage drop in the nanotube between the contacts [2]. Table 1 summarizes the results of fitting the data for a variety of devices. Meas. $\alpha$ refers to the value obtained directly from a least squares fit of the $G(T)$ curve. Scaled $\alpha$ refers to the value taken from the Luttinger scaling fit, as in Fig. 2. We see that, the values of $\alpha$ are indeed close to the predicted values for the two cases of $\alpha_{\text {end }}$ and $\alpha_{\text {bulk. }}$. However, we see a significant deviation for the measured and scaled values. This is most likely due to the appearance of the knee feature in the $R(T)$ data. Therefore, the scaled value is most likely the best measured value. Although we expect both cases of end and bulk tunneling to occur, from the scaled value, we can determine that the majority of tunneling is end tunneling. However, our values of scaled $\alpha$ are slightly higher than the predicted value for end tunneling. This can be attributed to a finite voltage drop in nanotube between the contacts and disorder in the MWCNTs. Based on our scaled $\alpha$, the Luttinger parameter, $g$ is estimated to be approaching the limit of what can be considered "less than 1" before it becomes "much, much less than 1". As $g$ decreases, it corresponds to increase in strength of electron-electron interactions. Therefore, our material can be judged to be just barely a LL and almost in the localized electron region of mesoscopic systems. We also note that the value of $\eta$ widely varies with some values above the predicted value and some well below the predicted value. This can be attributed to the coupling of the electrical contacts to the nanotube. In the case of very strong coupling (or very low contact resistance), the value of $\eta$ becomes significantly less than the predicted value.

There are other explanations for ZBAs in disordered conductors. For instance, it has been shown theoretically that in the case of high impedance contacts or transmission lines ZBA can occur [4-6]. This is obviously not the case for our devices, as we have very low contact resistances and the resistance of the nanotube is very small. However, other studies have also shown that a ZBA can occur in a disordered conductor due to effects associated with enhanced plasmon scattering $[5,19,20]$. It has been proposed that here $G(T) \sim e^{-\chi / \sqrt{T}}$, with $\chi$ being a measure of disorder [20]. We found, surprisingly, that these models did not fit our data as well as the Luttinger model. Although, based on the disorder in our nanotubes we cannot definitely conclude that there is LL behavior, it appears to be the most likely candidate.

Fig. 4 shows the temperature dependent MR for a hydrogen annealed and an argon-only annealed device. For the hydrogen annealed sample, the MR is positive at $6 \mathrm{~K}$. At higher temperatures, $\sim 15 \mathrm{~K}$ (for this sample) there is a transition to negative MR, which continues to remain negative for higher temperatures. This behavior was consistent for all samples studied. However, the transition temperature seems to vary from sample to sample, ranging from 


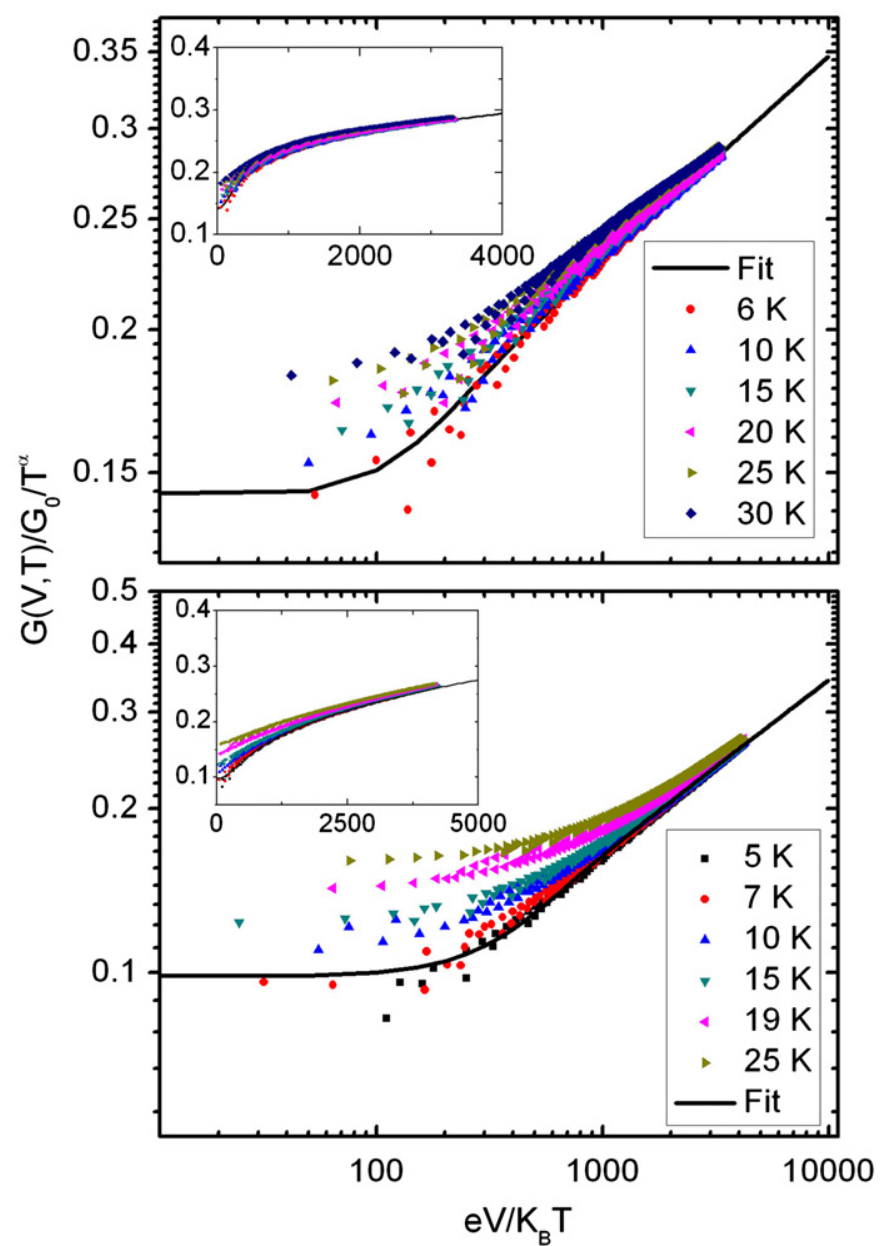

Fig. 3. Scaled conductivity versus $e V / k_{B} T$ for MWCNTs at various temperatures, representing Luttinger log-log scaling plots. The insets are not logarithmic. The upper graph shows data for an argon-only annealed device and the lower graph shows data for a hydrogen annealed device. For both devices, the MWCNTs were deposited for $75 \mathrm{~min}$.

$\sim 13$ to $40 \mathrm{~K}$. This confirms that the transition is not related to the observed knee in the $R(T)$ curves. Furthermore, the transition temperature was also found to be independent of inner diameter of the CNTs. In the case of the non-magnetic nanotubes, the MR is always positive for all tested samples.

The MR transition in the ferromagnetic nanotubes is most probably a result of an order-to-disorder transition as predicted by Bright [10]. Bright formulated a model for transport in disordered carbon structures when contemplating negative MR in different types of carbon fibers and filaments from the amorphous to the highly graphitized [10]. It was suggested that negative MR is the result of formation of Landau levels at the Fermi energy, which increases the density of states and the current path arising from disorder in the structure of the conductor. Taking into account several considerations for disordered carbon structures, it was shown that the MR of a disordered carbon structure in the presence of an applied field $B$ follows:

$\rho(B)=\frac{1+\mu^{2} B^{2}}{(n+p) e \mu\left[1+\mu^{2} B^{2}(p-n)^{2} /(p+n)^{2}\right]}$

Here, $p$ and $n$ are the particle and hole carrier densities, respectively, while $\mu$ is the carrier mobility. It is assumed that the application of the electric field does not affect the effective mass, and the scattering process or mobility. From Eq. (3) the MR ratio $\Delta \rho / \rho=(\rho(B)-\rho(0)) / \rho(0)$ can be calculated for two different limits.
First, if $n \ll p$, then the hole concentration is proportional to the density of states and

$\frac{\Delta \rho}{\rho} \sim-157 \mu^{4} B^{2}$

Here, values for the parameters were taken from Ref. [11]. This is the physical case of a very disordered nanotube. Second, when $|p-n| \ll p+n$, then

$\frac{\Delta \rho}{\rho} \sim\left(\frac{n_{0}+p_{0}}{n+p}-1\right)+\frac{n_{0}+p_{0}}{n+p} \mu^{2} B^{2}$

This is the case of a well-ordered nanotube. Here the $\mu^{2} B^{2}$ term dominates in high fields. Thus, the MR approaches that of a regular metallic conductor, for which $\Delta \rho / \rho=\mu^{2} B^{2}$.

In our disordered MWCNTs, at low temperatures and in the absence of a magnetic field, the device behaves like a LL, but only barely, as the disorder in the sample produces many electron-electron interactions that nearly make it a 2-dimensional system. However, upon application of a magnetic field the number of interactions increase significantly, especially at fields higher than $1 \mathrm{~T}$. This is evident in Fig. 4 that all the interesting behavior happens well above $1 \mathrm{~T}$, while for low fields it is effectively a straight line. However, despite increased interactions, at low temperatures the device behaves like a metal. As the temperature increases, thermal fluctuations introduce even more disorder into the system. Eventually, the MR transitions from the more ordered case to the disordered case become negative.

In the case of non-magnetic argon-only annealed devices, switching on a magnetic field will not significantly increase the number of electron-electron interactions because the necessary ferromagnetism is absent. Therefore, the device remains metallic and as the temperature increases, thermal fluctuations cause the mobility to decrease gradually.

On the other hand, there are at least two additional sources of scattering available in the hydrogen annealed nanotubes that are not present in the argon-only annealed nanotubes. We can rule out LL behavior because the field will destroy the quantum critical state of the LL. We can also rule out weak localization because weak localization is expected to be unobservable if ferromagnetism is present. In order to clarify this, we performed electrical measurements on samples fabricated for the application of a gate voltage and found no dependence on gate voltage. If the behavior was due to an Anderson transition we would expect the behavior of a gate voltage sweep to be different before and after the transition [21]. For a metal, we expect Coulomb blockade behavior leading to low temperature oscillations of a well-defined period [1]. For a semiconductor, we would expect the appearance of a minimum corresponding to the carrier minimum. However, we found that the behavior was roughly the same everywhere, showing irreproducible fluctuations. Thus, the device is most probably a metal and our measurement temperatures were not low enough to observe well-defined oscillations. This indicates that this is not a metal-insulator or metal-semiconductor type of transition. Fig. 4 shows the mobilities calculated as fitting parameters for the Bright model using Eqs. (4) and (5). However, we see the mobility of the hydrogen devices fall off more quickly at lower temperatures, which is a graphical representation of the Bright transition from order to disorder.

In conclusion, it is shown that at low temperatures, disordered multiwalled carbon nanotubes annealed in argon-only and in hydrogen exhibit a ZBA, which having explored several models, can be best fit by the Luttinger model. However, on the application of a magnetic field, negative magnetoresistance is observed only for the hydrogen annealed nanotubes that are ferromagnetic. This behavior is compatible with a Bright order-to-disorder transition arising from a combination of increased disorder due to electron-electron interactions, electron-magnon interactions, and phonon scattering. The argon-only annealed nanotubes do 

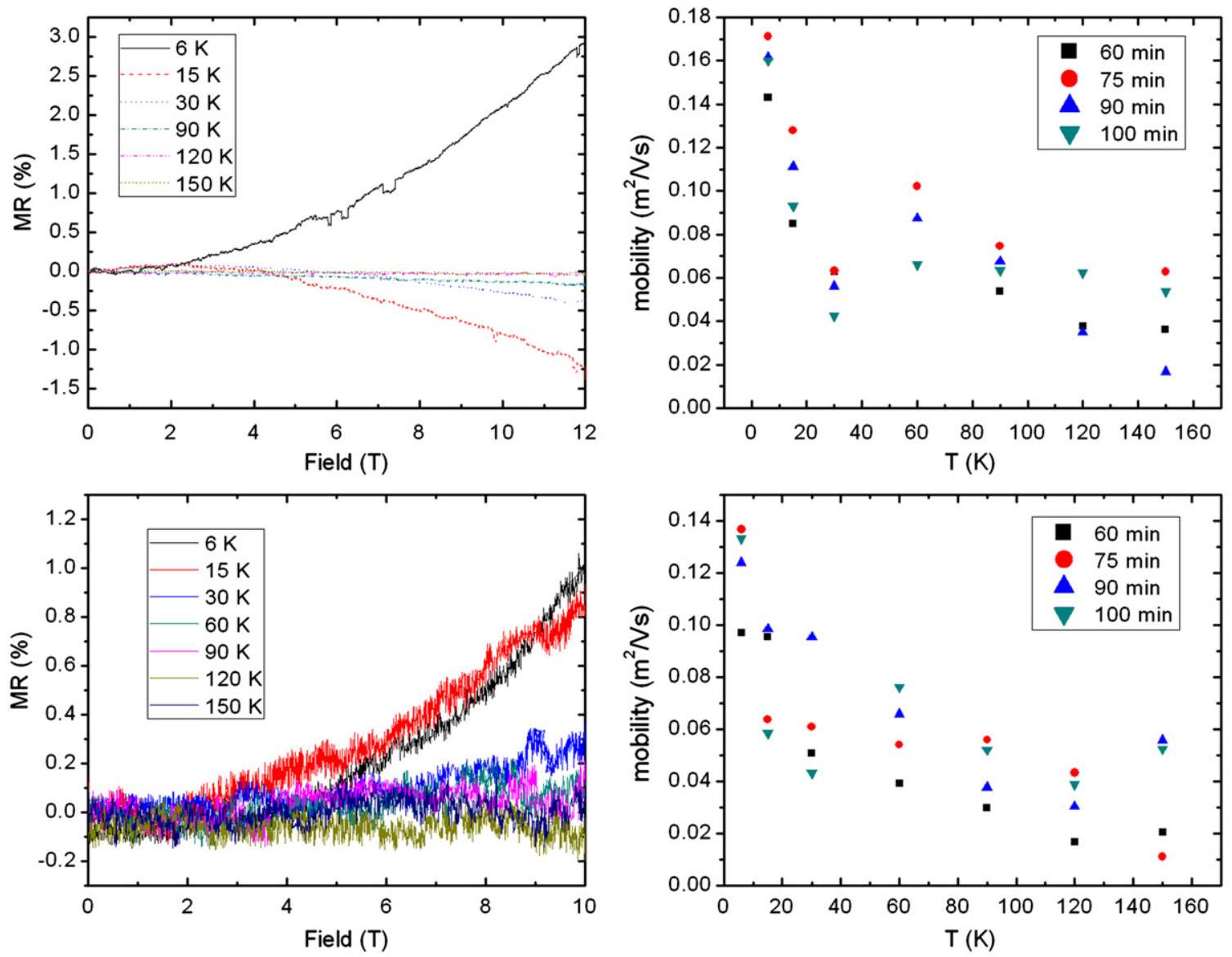

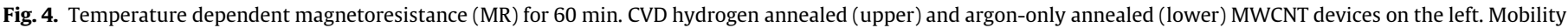
versus temperature for hydrogen annealed (upper) and argon-only annealed (lower) MWCNT devices calculated as parameters of the Bright model on the right.

not exhibit this transition because they lack several scattering pathways found in the hydrogen annealed nanotubes.

The authors acknowledge support from NSF CAREER Grant ECCS-0551468.

\section{References}

[1] Marc Bockrath, David H. Cobden, Jia Lu, Andrew G. Rinzler, Richard E. Smalley, Leon Balents, Paul McEuen, Nature 397 (1999) 598.

[2] E. Graugnard, P.J. de Pablo, B. Walsh, A.W. Ghosh, S. Datta, R. Reifenberger, Phys Rev. B 64 (2001) 125407.

[3] Vikram V. Deshpande, Marc Bockrath, Leonid I. Glazman, Amir Yacoby, Nature 464 (2010) 209.

[4] S.M. Girvin, L.I. Glazman, M. Jonson, D.R. Penn, M.D. Stiles, Phys. Rev. Lett. 64 (1990) 3183.

[5] A.M. Rudin, I.L. Aleiner, L.I. Glazman, Phys. Rev. B 55 (1997) 9322.

[6] M.H. Devoret, D. Esteve, H. Grabert, G.-L. Ingold, H. Pothier, C. Urbina, Phys. Rev. Lett. 64 (1824) 1990.

[7] Reeta Tarkiainen, Marcus Ahlskog, Pertti Hakonen, Mikko Paalanen, Physica E 18 (2003) 206.

[8] Reeta Tarkiainen, Marcus Ahlskog, A. Zyuzin, Pertti Hakonen, Mikko Paalanen, Phy. Rev. B 69 (2004) 033402.
[9] R. Saito, G. Dresselhaus, M.S. Dresselhaus, Physical Properties of Carbon Nanotubes, Imperial College Press, Singapore, 1998.

[10] A.A. Bright, Phys. Rev. B 20 (1979) 5412.

[11] M.S. Dresselhaus, G. Dresselhaus, K. Sugihara, I.L. Spain, H.A. Goldberg Graphite Fibers and Filaments, Springer, New York, 1988

[12] A.L. Friedman, Hyunkyung Chun, Yung Joon Jung, Don Heiman, E.R. Glaser, Latika Menon, Phys. Rev. B 81 (2010) 115461.

[13] P. Esquinazi, D. Spemann, R. Hohne, A. Setzer, K.-H. Han, T. Butz, Phys. Rev. Lett. 91 (2003) 227201.

[14] Y. Ma, P.O. Lehtinen, A.S. Foster, R.M. Nieminen, Phys. Rev. B 72 (2005) 085451.

[15] S. Talapatra, P.G. Ganesan, T. Kim, R. Vajtai, M. Huang, M. Shima, G. Ramanath, D. Srivastava, S.C. Deevi, P.M. Ajayan, Phys. Rev. Lett. 95 (2005) 097201.

[16] Y. Zhang, S. Talapatra, S. Kar, R. Vajtai, S.K. Nayak, P.M. Ajayan, Phys. Rev. Lett. 99 (2007) 107201.

[17] A.L. Friedman, Derrick Brittain, Latika Menon, J. Chem. Phys. 127 (2007) 154717 references contained therein..

[18] Hongwei Xuesong Li, Lijie Zhu, Cailu Ci, Zongqiang Xu, Bingquig Mao, Ji Wei, Liang, Dehai Wu, Carbon 39 (2001) 2077.

[19] W. Yi, L. Hu, H. Hu, Z.W. Pan, S.S. Xie, Phys. Rev. Lett. 91 (2003) 076801.

[20] V. Krstic, S. Blumentritt, J. Muster, S. Roth, A. Rubio, Phys. Rev. B 67 (R) (2003) 041401.

[21] P.P. Edwards, R.L. Johnston, C.N.R. Rao, D.P. Tunstall, F. Hensel, Phil. Trans. R. Soc. Lond. A 356 (1998) 5. 